\title{
Treatment with Non-specific HDAC Inhibitors Administered after Disease Onset does not Delay Evolution in a Mouse Model of Progressive Multiple Sclerosis
}

\author{
Daniela Buonvicino, ${ }^{* \dagger}$ Giuseppe Ranieri ${ }^{\dagger}$ and Alberto Chiarugi \\ Department of Health Sciences, Section of Clinical Pharmacology and Oncology, University of Florence, Florence, Italy
}

\begin{abstract}
Drugs able to efficiently counteract progression of multiple sclerosis (MS) are still an unmet need. Several lines of evidence indicate that histone deacetylase inhibitors (HDACi) are clinically-available epigenetic drugs that might be repurposed for immunosuppression in MS therapy. Here, we studied the effects of HDACi on disease evolution in myelin oligodendrocyte glycoprotein (MOG)-immunized NOD mice, an experimental model of progressive experimental autoimmune encephalomyelitis (PEAE). To obtain data of potential clinical relevance, the HDACi panobinostat, givinostat and entinostat were administered orally adopting a daily treatment protocol after disease onset. We report that the 3 drugs efficiently reduced in vitro lymphocyte proliferation in a dosedependent manner. Notably, however, none of the drugs delayed evolution of PEAE or reduced lethality in NOD mice. In striking contrast with this, however, the lymphocyte proliferation response to MOG as well as Th1 and Th17 spinal cord infiltrates were significantly lower in animals exposed to the HDACi compared to those receiving vehicle. When put into a clinical context, for the first time data cast doubt on the relevance of HDACi to treatment of progressive MS (PMS). Also, our findings further indicate that, akin to PMS, neuropathogensis of PEAE in NOD mice becomes independent from autoimmunity, thereby corroborating the relevance of this model to experimental PMS research. (c) 2021 IBRO. Published by Elsevier Ltd. All rights reserved.
\end{abstract}

Key words: progressive EAE, HDAC inhibitor, panobinostat, entinostat, givinostat.

\section{INTRODUCTION}

Multiple sclerosis (MS) is a polygenic neuroimmune disorder whose pathogenesis is characterized by an autoimmune and neurodegenerative component. It affects about 2.3 million people worldwide and is recognized as the leading cause of disability among young individuals (Thompson et al., 2018). From a pathophysiological perspective, a complex interplay between adaptive and innate immune responses against myelin recently emerged as central to disease pathogenesis (Kutzelnigg and Lassmann, 2014). Accordingly, immunoregulatory drugs targeting different events of the autoimmune response proved of therapeutic relevance and significantly contributed to expand the therapeutic armamentarium available to counteract MS (Ontaneda et al., 2019). In this light, however, it is worth noting that

\footnotetext{
*Corresponding author. Address: Department of Health Sciences, Section of Clinical Pharmacology and Oncology, University of Florence, Viale Pieraccini 6, 50139 Firenze, Italy.

E-mail address: daniela.buonvicino@unifi.it (D. Buonvicino).

These authors equally contributed to this work. Abbreviations: HDACi, histone deacetylase inhibitors; MOG, myelin oligodendrocyte glycoprotein; MS, multiple sclerosis; PEAE, progressive experimental autoimmune encephalomyelitis; PMS, progressive MS
}

the major therapeutic achievements relate to treatment of the relapsing-remitting (RR) form of MS. Conversely, in spite of the recent approval of ocrelizumab (Montalban et al., 2017) and siponimod (Kappos et al., 2018) for the treatment of progressive MS (PMS), the latter is still unsatisfactory and new drugs able to efficiently counteract molecular and cellular mechanisms of disease progression are urgently needed. In this regard, it is well known that in the multistep process of drug development, repurposing of compounds already approved for the treatment of different disorders significantly expedites clinical development. In an attempt to apply drug repurposing to MS therapy, several lines of evidence suggest that inhibitors of histone deacetylases (HDACi) may represent an innovative therapeutic strategy (Gray and Dangond, 2006; Peedicayil, 2016). HDACs belong to a class of 11 enzymes involved in epigenetic regulation of chromatin architecture and gene expression, and HDACi represent the first class of epigenetic drugs that reached the clinical arena for treatment of different hematological malignancies (Ceccacci and Minucci, 2016; Cappellacci et al., 2020). Additional trials with HDACi are currently ongoing in patients affected by multiple types of disorders, such as glioblastoma, Duchenne muscular dystrophy and myelofibrosis (Galanis et al., 2009; Friday et al., 2012; 
Bettica et al., 2016; Mascarenhas et al., 2020; Wössner et al., 2020). As far as MS is concerned, numerous studies report the potent immunosuppressive and antiinflammatory effects of HDACi in in vitro and in vivo models of innate or adaptive immune activation (Moreira et al., 2003; Camelo et al., 2005; Faraco et al., 2009; Bagchi et al., 2018; Jayaraman et al., 2018; Lillico et al., 2018; Sun et al., 2018). These findings, along with evidence that HDACi also target a plethora of molecular mechanisms underpinning both neuron and oligodendrocyte cell death, further strengthen the rationale of their use in MS therapy (Faraco et al., 2011; KhorshidAhmad et al., 2016). In keeping with this, epigenetic modulation of transcriptional homeostasis with HDACi in rodents affected by experimental autoimmune encephalomyelitis prompts immunosuppression and lessens disease severity (Jayaraman et al., 2017; Jayaraman et al., 2018). Although these findings corroborate the therapeutic potential of HDAC inhibitors in MS therapy, it is worth noting that they have been administered adopting a clinically-irrelevant, treatment schedule.

We have recently characterized the mouse model of primary progressive EAE (PEAE) in NOD mice from an immunological and neuropathological perspective. Of note, in keeping with data in PMS patients, we report that PEAE evolution in NOD mice is insensitive to immunosuppression and featured by early mitochondrial dysfunction (Buonvicino et al., 2019). The reliability of this EAE model to PMS research is emphasized by the recent evidence of inefficacy of high dose biotin in PMS patients (Cree et al., 2020), a negative finding already predicted by our study in PEAE NOD mice (Buonvicino et al., 2019). In the present study, to further understand the pathogenetic relevance of the neuroimmune response in a model of progressive MS, we studied the effects of different HDACi on disease progression in PEAE NOD mice. We report that, at variance with prior work, none of the HDACi with different degrees of selectivity towards HDAC isoforms suffices to counteract disease progression in spite of evidence for concomitant immunosuppression.

\section{EXPERIMENTAL PROCEDURES}

\section{EAE induction and treatment}

All animal care and experimental procedures were performed according to the European Community guidelines for animal care (European Communities Council Directive 2010/63/EU) and were approved by the Committee for Animal Care and Experimental Use of the University of Florence. Female NOD/ShiLtj mice (Charles River, Milan, Italy) were housed in a conventional unit (5-6 per cage) with free access to food and water, and maintained on a $12 \mathrm{~h}$ light/dark cycle at $21^{\circ} \mathrm{C}$ room temperature. EAE was induced in 10 week-old NOD/ShiLtj mice with $\mathrm{MOG}_{35-55}$ peptide (synthesized by EspiKem Srl., University of Florence, Italy) as reported (Buonvicino et al., 2019). Clinical signs of EAE were examined daily by blinded operators. The following score were assigned: 0 , normal; 0.25 or 0.5 , splay reflex test (performed by lifting the mouse by its tail and observing the degree of hindlimb splay during $10 \mathrm{~s}$. If both hindlimbs were splayed outward away from the abdomen, a 0 score was assigned. If one or both hindlimbs were partially retracted toward the abdomen without touching it, a score 0.25 or 0.5 was assigned, respectively); 1 , weakness of the tail/hind limbs; 2, ataxia and/or difficulty in righting; 3 , paralysis of the hind limbs and/or paresis of the forelimbs. Because of ethical reasons, score 3 mice were euthanized as soon as they reached the score. Panobinostat, entinostat and givinostat (MedChem Express, USA) were dissolved in PBS and orally administrated on a daily basis at $10 \mathrm{mg} / \mathrm{kg}$ body weight for entinostat and givinostat, and $20 \mathrm{mg} / \mathrm{kg}$ body weight for panobinostat. Treatments started from score 1. Immunized vehicle-treated animals daily received the same amount of PBS.

\section{Cell death analysis}

Cell death was quantified by means of cytofluorometric analysis. Cells extracted from spleen $\mathrm{MOG}_{35-55^{-}}$ immunized mice at disease onset (day 30 post immunization) were cultured in complete RPMI in 96 wells plates $\left(2 \times 10^{5}\right.$ cells per well) and stimulated with MOG $_{35-55}(20 \mu \mathrm{g} / \mathrm{ml})$ in presence or not of different concentrations of panobinostat, entinostat and givinostat. After $72 \mathrm{~h}$, Briefly, cells were exposed to $3 \mu \mathrm{g} / \mathrm{ml}$ propidium iodide (Sigma Aldrich, Milan, Italy) incubated at $37^{\circ} \mathrm{C}$ for $10 \mathrm{~min}$, and then analyzed by the flow cytometer Coulter EPICS XL equipped with the EXPO32 Flow Cytometry ADC software (Buonvicino et al., 2013).

\section{Lymphocytes proliferation}

Cells extracted from spleen $\mathrm{MOG}_{35-55}$-immunized mice at disease onset (day 30 post immunization) were cultured in complete RPMI in 96 wells plates $\left(2 \times 10^{5}\right.$ cells per well) and stimulated with $\mathrm{MOG}_{35-55}(20 \mu \mathrm{g} / \mathrm{ml})$ in presence or not of different concentrations of panobinostat, entinostat and givinostat. After $72 \mathrm{~h}$, the proliferative response was measured by $\left[{ }^{3} \mathrm{H}\right]$ thymidine incorporation test as reported (Buonvicino et al., 2019). Similarly, cells extracted from spleen $\mathrm{MOG}_{35-55^{-}}$ immunized mice treated or not 30 day with panobinostat (20 mg/kg oral, daily), entinostat (10 mg/kg oral, daily) or givinostat $(10 \mathrm{mg} / \mathrm{kg}$ oral, daily) were cultured in complete RPMI in 96 wells plates $\left(2 \times 10^{5}\right.$ cells per well) and stimulated with $\mathrm{MOG}_{35-55}(20 \mu \mathrm{g} / \mathrm{ml})$. The proliferative response was measured as described above.

\section{Quantitative PCR}

Total RNA was isolated from spinal cord of NODimmunized mice using Trizol Reagent (Life Technologies). One $\mu \mathrm{g}$ of RNA was retrotranscribed using iScript (Bio-Rad, Milan, Italy). RT-PCR was performed as reported (Buonvicino et al., 2018). The following primers were used: T-bet: forward 5'- TGCCTACCAGAACGCAGAGATCACTC $-3^{\prime}$ and reverse $5^{\prime}-$ GTAGAAACGGCTGGGAACAGGATACTG -3'; ROR $\gamma t$ : forward 5' - CCATTCAGTATGTGGTGGAGTTTGCCAA $3^{\prime}$ and reverse $5^{\prime}$ - GCAGCCCAAGGCTCGAAACAGCT - 
3'; GATA3: forward 5'- TGCCTGTGGGCTGTACTACAAGCTTCA -3' and reverse 5'- GATGTGGCTCAGGGATGACATGTGTC -3'; Foxp3: forward $5^{\prime}$ CAAGCAGATCATCTCCTGGAT $-3^{\prime}$ and reverse $5^{\prime}-$ GTGGCTACGATGCAGCAAGAG -3'; 18S: forward 5'-A AAACCAACCCGGTGAGCTCCCTC $-3^{\prime}$ and reverse $5^{\prime}$ CTCAGGCTCCCTCTCCGGAATCG - $3^{\prime}$.

\section{Statistical analysis}

Data are expressed as mean \pm SEM or with median and 5th-95th percentile. In order to evaluate difference in continuous parameters between two groups MannWhitney test (according to Kolmogorov-Smirnov test for normality) is used. To test the difference between more than two groups ANOVA plus Tukey's (according to Kolmogorov-Smirnov test for normality) are used. The differences in overall survival between groups are tested using Kaplan-Meier curve. Differences were considered to be significant when $p$-value $<0.05$. Statistical analyses were carried out using GraphPad Prism (version 7).

\section{RESULTS}

\section{Effect of different HDACi on the recall response of lymphocytes from MOG-immunized NOD mice}

As mentioned above, several studies evaluated the effects of HDACi in mice with EAE (Camelo et al., 2005; Jayaraman et al., 2017; Jayaraman et al., 2018). Of note, the tested compounds are non-specific inhibitors of the different HDAC isoforms (panHDACi). Further, they were not compounds that reached the clinical development or approval for therapeutic purposes. Hence, in order to better identify the therapeutic potential of HDACi in PMS therapy, we planned to test multiple compounds with a realistic translational potential to MS patients. To this end, among the numerous inhibitors available, we selected panobinostat, entinostat and givinostat (Table 1). The first is an ultrapotent panHDACi approved for treatment of resistant multiple myeloma (Laubach et al., 2015). The second is not yet clinically available but shows selectivity towards Class I HDAC1, -2 and -3 , the most functionally-relevant nuclear HDACs, leaving unaffected the Class II cytosolic isoforms (Connolly et al., 2017). Finally, the third is also a pan inhibitor (although shows partial selectivity towards class I and II) but, at variance with panobinostat, shows a more favorable tolerability profile and is currently under development for a neuromuscular disorders (Bettica et al., 2016). We first wondered whether the compounds were indeed able to prevent proliferation of autoreactive lymphocytes. As expected, splenocytes derived form MOG-immunized mice at disease onset (day 30 post immunization) underwent extensive proliferation when re-challenged in vitro with MOG (Fig. 1A). Notably, exposure of cultured lymphocytes to the different HDAC inhibitors caused a concentration dependent inhibition of proliferation, in keeping with the immunosuppressive effects of HDACi. Even though panobinostat is reported to be more potent HDACi than entinostat and givinostat (Singh et al.,
2018), we found that the $\mathrm{IC}_{50}$ S on MOG-dependent lymphocyte proliferation did not differ among the 3 compounds (Fig. 1A). Although this apparent inconsistency might be reconciled with the low permeability of panobinostat through the plasmamembrane, we ruled out a possible non-specific cytotoxic effects of the drugs by means of PI staining. Data demonstrated that in the adopted concentration range of $1-100 \mathrm{nM}$, none of the tested HDACi prompted cytotoxicity upon a $72 \mathrm{~h}$ incubation (Fig. 1B). Taken together, these findings indicate that HDACi efficiently suppress proliferation of autoreactive lymphocytes, a key pathogenetic event during initial CNS infiltration and the ensuing waves sustained by epitope spreading.

\section{Treatment with different HDACi administered after disease onset does not affect disease progression in PEAE NOD mice}

In light of the ability of the $3 \mathrm{HDACi}$ to counteract proliferation of autoreactive lymphocytes, we then investigated their effects on EAE progression in NOD mice. To better appreciate the therapeutic potential of this class of drugs, we designed a clinically-relevant treatment schedule consisting in a daily and oral exposure to the drugs beginning $24 \mathrm{~h}$ after reaching score 1. As previously reported, in this EAE model onset occurs around day 25 with a variability ranging from day 19 to 34 . Unexpectedly, none of the $3 \mathrm{HDACi}$ had any effect on the temporal kinetics of disease progression, with vehicle- and drug-treated mice reaching concomitantly the same severity of neurological impairment (Figs. 2A-C and S1-3). Reportedly, PEAE invariantly prompts lethality in NOD mice. In this regard, we found that none of the groups of mice exposed to the HDACi showed an extended survival compared to vehicle-treated animals (Fig. 2DF). To rule out possible toxic effects of the compounds during treatment of PAE NOD mice, we evaluated the animals' body weight at day 30 after treatment and found no differences with animals receiving vehicle (Fig. 2G).

\section{Effects of HDACi on neuroimmune infiltrates in PEAE NOD mice}

The ability of HDACi to reduce proliferation of autoreactive lymphocytes in vitro was at odds with their lack of effects on PEAE progression. This inconsistency prompted us to evaluate whether the 3 drugs were indeed able to suppress the autoimmune response in MOG-immunized NOD mice. As expected, rechallenge of splenocytes of PEAE NOD mice with MOG prompted rapid proliferation. However, the proliferation response was significantly reduced when splenocytes were harvested from animals exposed to HDACi (Fig. 3A). These findings are in keeping with the ability of the compounds to impair proliferation of MOG-specific lymphocytes when directly added to the culture medium (see Fig. 1A). Further corroborating the ability of $\mathrm{HDACi}$ to prompt immunosuppression in PEAE NOD mice, we also found that panobinostat, entinostat and givinostat 
Table 1. HDAC inhibition ( $\mathrm{IC}_{50}$ in $\left.\mathrm{nM}\right)$ by panobinostat, entinostat and givinostat

\begin{tabular}{|c|c|c|c|}
\hline HDAC isoforms & Panobinostat $^{\mathrm{a}}$ & Entinostat $^{\mathrm{b}}$ & Givinostat $^{\mathrm{C}}$ \\
\hline HDAC1 & 2.5 & 163 & 198 \\
\hline HDAC2 & 13.2 & 396 & 325 \\
\hline HDAC3 & 2.1 & 605 & 157 \\
\hline HDAC4 & 203 & $>2000$ & 854 \\
\hline HDAC5 & 7.8 & $>2000$ & 1059 \\
\hline HDAC6 & 10.5 & 3930 & 532 \\
\hline HDAC7 & 531 & $>2000$ & 524 \\
\hline HDAC8 & 277 & $>10000$ & 541 \\
\hline HDAC9 & 5.7 & $>2000$ & 315 \\
\hline HDAC10 & 2.3 & $>2000$ & 340 \\
\hline HDAC11 & 2.7 & NA & 292 \\
\hline
\end{tabular}

NA: not available.

a Ref. (Atadja, 2009).

b Ref. (Cai et al., 2015; Xie et al., 2018).

c Ref. (Li et al., 2015).
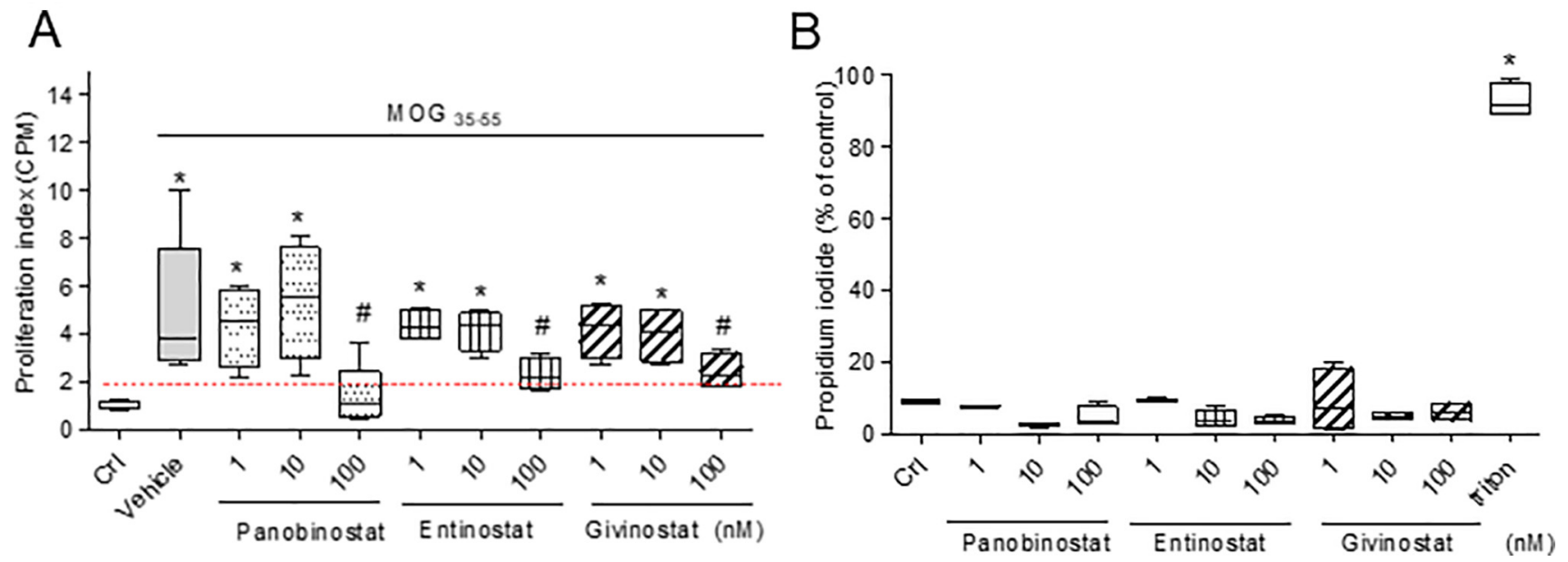

Fig. 1. Effects of different HDAC inhibitors on lymphocyte proliferation and viability. (A) Proliferation of spleen lymphocytes from $\mathrm{MOG}_{35-55^{-}}$ immunized NOD mice harvested at disease onset were re-challenged in vitro with $\mathrm{MOG}_{35-55}(20 \mu \mathrm{g} / \mathrm{ml})$ in the presence or absence of different concentrations of panobinostat, entinostat and givinostat. (B) Propidium iodide positive splenocytes treated in vitro with different concentrations of panobinostat, entinostat or givinostat for $72 \mathrm{~h}$. In (A) and (B) each column is the mean \pm SEM of at least 3 animals. ${ }^{*} p<0.05 \mathrm{vs}$ Crl, \#p<0.05 vs Vehicle (ANOVA plus Tukey's test).

reduced the transcript levels for Tbet, a classic marker of Th1 cells, in the lumbar spinal cord of score 3 PEAE NOD mice (Fig. 3B). Similarly, transcript levels for the Th17 cell marker ROR- $\gamma$ were lower in the spinal cord of PEAE mice exposed to HDACi compared to those receiving vehicle (Fig. 3C). Conversely, panobinostat, entinostat or givinostat did not alter spinal cord transcripts for the Th2 cell marker GATA3 or the Treg marker Foxp3 (Fig. 3D, E).

\section{DISCUSSION}

In the present study, we provide the first evidence that treatment with HDAC inhibitors administered after disease onset does not reduce disease severity in a mouse model of progressive MS. Remarkably, we also show that lack of effects on progression of EAE occurs in spite of the ability of these drugs to counteract the MOG-specific autoimmune response. These findings, together with prior evidence that dexamethasone reduces the neuroimmune response but is also unable to counteract disease evolution in NOD mice with PEAE, emphasize the relevance of this animal model to PMS research. Indeed, the insensitivity to immunosuppressants of EAE progression in NOD mice somehow recapitulates the inefficacy of these drugs in PMS patients.

It is worth noting that in the present study we separately evaluated the effects of 3 different and potent HDAC inhibitors obtaining exactly the same results. On the one hand, this corroborates the reliability of our findings, and on the other strengthens our conclusion on 
A

B
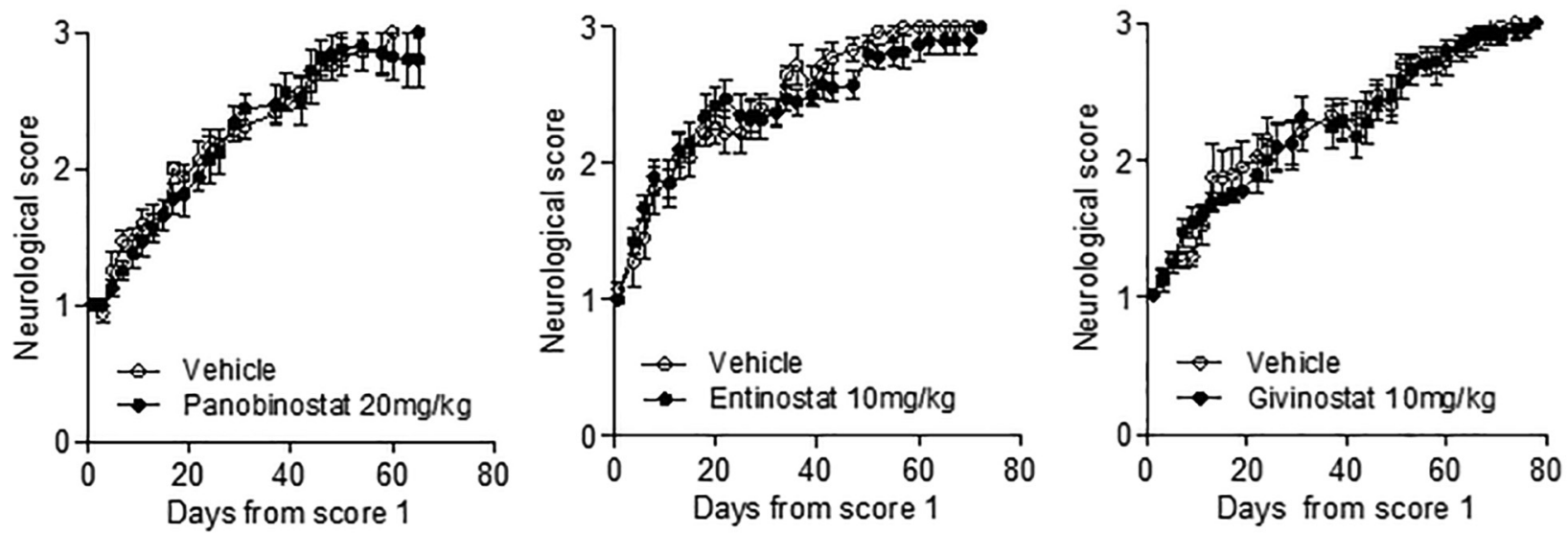

D E
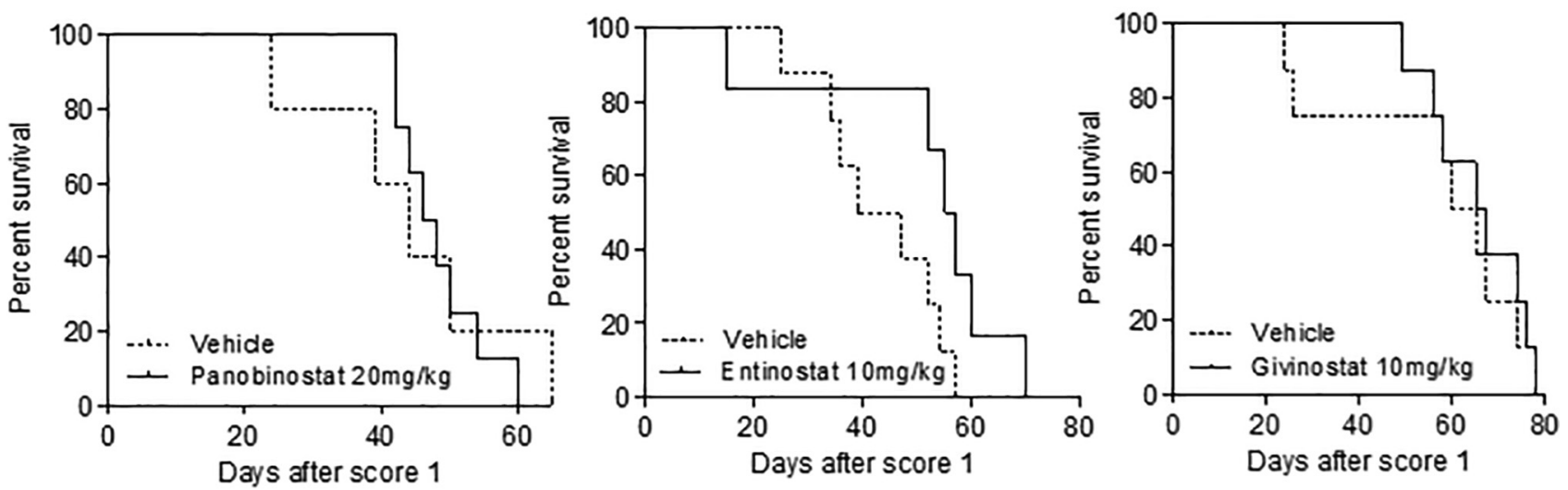

G

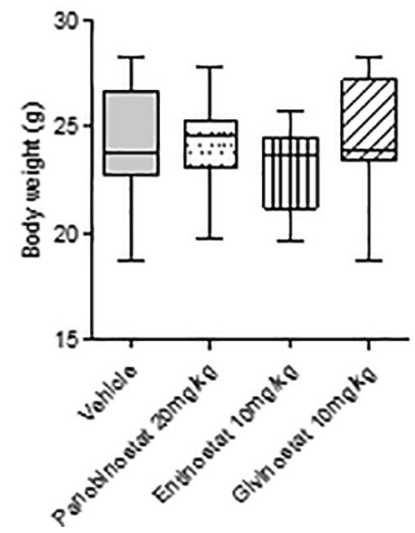

Fig. 2. Effects of HDAC inhibitors administered after disease onset on disease progression in PEAE NOD mice. Effects of daily, oral treatment from score 1 with panobinostat $(20 \mathrm{mg} / \mathrm{kg})$, entinostat $(10 \mathrm{mg} / \mathrm{kg})$ and givinostat $(10 \mathrm{mg} / \mathrm{kg})$ on neurological score (A) $(n=10 \mathrm{mice}$ per group; Mann Whitney test), survival (B) ( $n=10$ mice per group; Kaplan-Meier), and body weight (C) ( $n=10$ mice per group; ANOVA plus Tukey's test) in PEAE NOD mice.

the possible inefficacy of HDAC inhibition as drugs useful to counteract PMS. Also, our data appear of considerable significance in light of the interest for testing clinically- available HDACi in PMS patients. In this regard it is worth noting that we adopted a post onset-treatment paradigm starting drug exposure from score 1. 

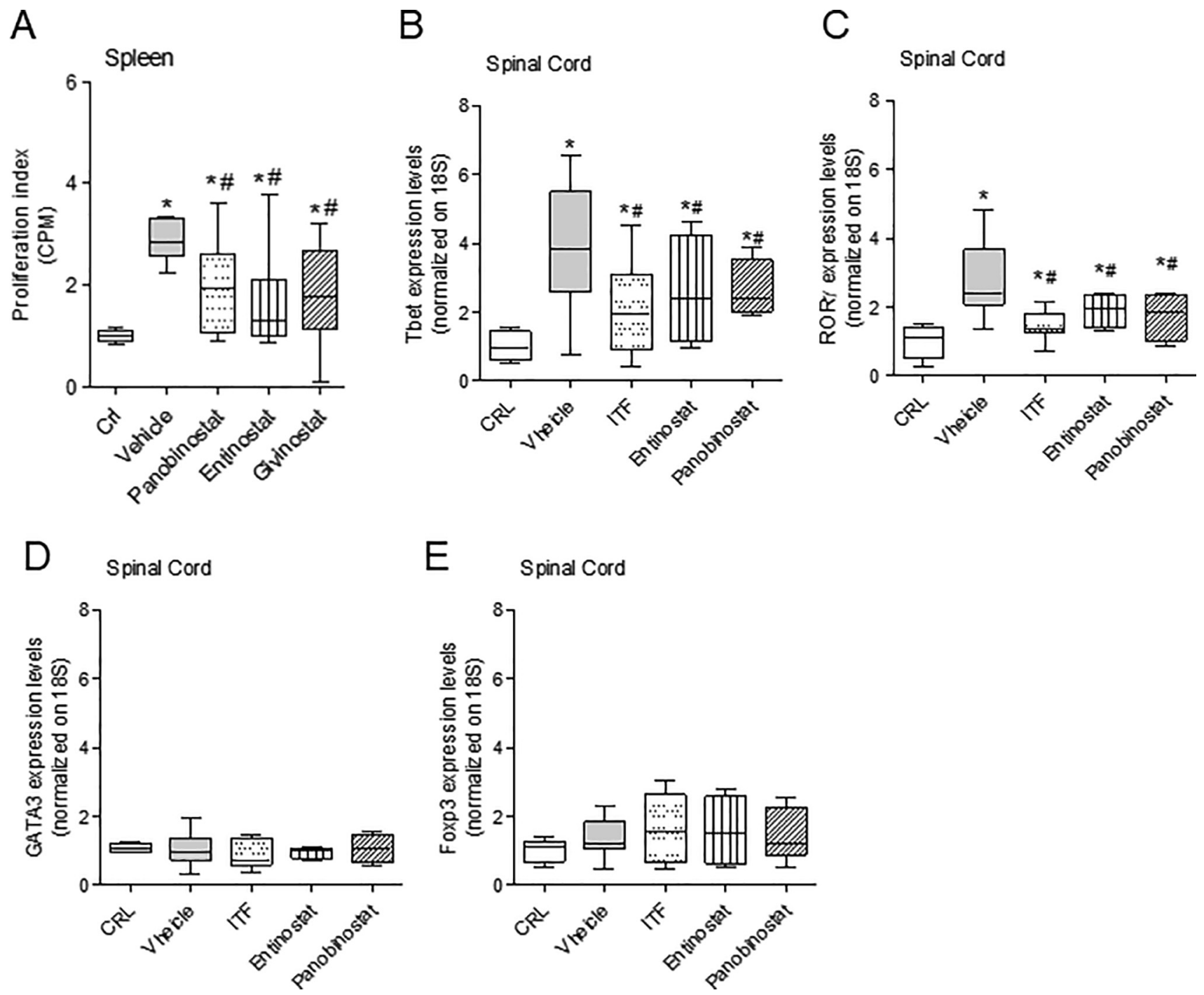

Fig. 3. Effects of HDAC inhibitors on lymphocyte MOG sensitization and spinal cord infiltration in PEAE NOD mice. (A) Proliferation of splenocytes from score $3 \mathrm{MOG}_{35-55}$-immunized NOD mice treated or not with panobinostat, entinostat and givinostat and re-challenged in vitro with $\mathrm{MOG}_{35-55}$. Effects of panobinostat, entinostat and givinostat on expression of Tbet ${ }^{+}-$Th1 (B), ROR yt ${ }^{+}$-Th17 (C), GATA3 ${ }^{+}$-Th2 (D) and Foxp3 ${ }^{+}$-Treg (E) cells in the spinal cord of score 3 PEAE mice. Each point/column represents the mean \pm SEM of at least 5 animals per group. ${ }^{*} p<0.05$ vs $\mathrm{Crl}$, $\# p<0.05$ vs Vehicle (ANOVA plus Tukey's test).

Inevitably, this reduces the overall immunosuppressant impact of the 3 compounds during MOG sensitization, but at the same time represents a clinically-relevant treatment protocol. Our negative findings, along with evidence that HDACi affords protection from EAE when administered in a prophylactic paradigm ( $\mathrm{Ge}$ et al., 2013; Jayaraman et al., 2018), suggest that during PEAE in NOD mice immune-independent processes take over the initial encephalitogenic response against MOG. Possibly, neurodegenerative events including virtual-hypoxia insults leading to axonal degeneration (Trapp and Stys, 2009), as well as a shift form T cell-responses to neurotoxic glial cell activation (Weiner, 2008) may render PEAE insensitive to immunosuppression. In principle, inefficacy of HDACi might be due to low doses or pharmacokinetic issues related to the intrinsic bioavailability of the 3 selected compounds. In this regard, it is worth noting that the doses we adopted in vivo are consistent to those pre- viously used in studies showing efficacy of the compounds on multiple parameters (Ocio et al., 2010; Regna et al., 2014; Orillion et al., 2017). More importantly, our findings indicating that exposure to the $3 \mathrm{HDACi}$ reduced MOG sensitization in NOD mice as well as their Th1 and Th17 spinal cord infiltrates are clear evidence of pharmacodynamic activity and rule out the possibility of inefficacy due to low doses. These considerations taken together, therefore, further corroborate evidence that the pathogenesis of progression in PEAE NOD mice becomes independent from the underlying autoimmune response upon disease onset. It is also worth noting that none of the HDACi showed even a tendency to reduce severity and progression rate of PEAE in spite of their ability to reduce the neuroimmune response. We reason, therefore, that the processes underpinning progression become entirely independent form the autoimmune response. 
Overall, data are consistent with evidence of early mitochondrial impairment and ensuing widespread neurodegeneration in the spinal cord of PEAE NOD mice (Buonvicino et al., 2019), a cascade of events that appears of key pathogenetic relevance but intrinsically insensitive to inhibition of HDACs. This interpretation is also in keeping with our recent study showing the ability of dexpramipexole, a compound able to sustain mitochondrial bioenergetics, to reduce neurodegeneration and delay progression in NOD mice with PEAE (Buonvicino et al., 2020). We confirm the ability of HDACi to counteract autoreactive lymphocyte proliferation as well as the neuroimmune infiltrates in the course of autoimmune encephalomyelitis in mice. These pharmacodynamic effects, however, appears unable to counteract EAE progression if prompted after disease onset. Prior work showing the key role of specific HDACs such as isoforms 1 (Göschl et al., 2018), 6 (LoPresti, 2019) and 11 (Sun et al., 2018) in non-progressive EAE models further corroborates the hypothesis that epigenetics plays a different role in relapsing-remitting and progressive EAE and possibly MS. Overall, data are in keeping with the general inefficacy of immunosuppressants in patients affected by MS progression, and provide the first hint that epigenetic therapy with HDACi is inefficacious when put into the clinical context of primary progressive MS that inevitably precludes preventive treatments.

\section{CONFLICT OF INTEREST}

Authors declare no conflict of interest.

\section{ACKNOWLEDGMENT}

This work was supported by grants from Italian Foundation for Multiple Sclerosis 2014/R/6 (recipient AC).

\section{AUTHOR CONTRIBUTIONS}

A.C., D.B. and G.R designed the experiments; D.B. and G.R. carried out in vivo experiments; D.B. carried out in vitro experiments; D.B. and G.R. performed the statistical analysis; A.C. wrote the paper. All authors reviewed the manuscript.

\section{REFERENCES}

Atadja P (2009) Development of the pan-DAC inhibitor panobinostat (LBH589): successes and challenges. Cancer Lett 280:233-241. Bagchi RA, Ferguson BS, Stratton MS, Hu T, Cavasin MA, Sun L, Lin Y-H, Liu D, et al. (2018) HDAC11 suppresses the thermogenic program of adipose tissue via BRD2. JCl Insight 3 e120159.

Bettica P, Petrini S, D'Oria V, D'Amico A, Catteruccia M, Pane M, Sivo S, Magri F, et al. (2016) Histological effects of givinostat in boys with Duchenne muscular dystrophy. Neuromuscul Disord 26:643-649.

Buonvicino D, Formentini L, Cipriani G, Chiarugi A (2013) Glucose deprivation converts poly(ADP-ribose) polymerase-1 hyperactivation into a transient energy-producing process. J Biol Chem 288:36530-36537.

Buonvicino D, Ranieri G, Pratesi S, Gerace E, Muzzi M, Guasti D, Tofani L, Chiarugi A (2020) Neuroprotection induced by dexpramipexole delays disease progression in a mouse model of progressive multiple sclerosis. Br J Pharmacol 177:3342-3356.
Buonvicino D, Ranieri G, Pratesi S, Guasti D, Chiarugi A (2019) Neuroimmunological characterization of a mouse model of primary progressive experimental autoimmune encephalomyelitis and effects of immunosuppressive or neuroprotective strategies on disease evolution. Exp Neurol 322 113065.

Buonvicino D, Urru M, Muzzi M, Ranieri G, Luceri C, Oteri C, Lapucci A, Chiarugi A (2018) Trigeminal ganglion transcriptome analysis in 2 rat models of medication-overuse headache reveals coherent and widespread induction of pronociceptive gene expression patterns. Pain 159:1980-1988.

Cai J, Wei H, Hong KH, Wu X, Cao M, Zong X, Li L, Sun C, et al. (2015) Discovery and preliminary evaluation of 2aminobenzamide and hydroxamate derivatives containing 1,2,4oxadiazole moiety as potent histone deacetylase inhibitors. Eur $\mathrm{J}$ Med Chem 96:1-13.

Camelo S, Iglesias AH, Hwang D, Due B, Ryu H, Smith K, Gray SG, Imitola J, et al. (2005) Transcriptional therapy with the histone deacetylase inhibitor trichostatin A ameliorates experimental autoimmune encephalomyelitis. J Neuroimmunol 164:10-21.

Cappellacci L, Perinelli DR, Maggi F, Grifantini M, Petrelli R (2020) Recent progress in histone deacetylase inhibitors as anticancer agents. Curr Med Chem 27:2449-2493.

Ceccacci E, Minucci S (2016) Inhibition of histone deacetylases in cancer therapy: lessons from leukaemia. $\mathrm{Br} J$ Cancer 114:605-611.

Connolly RM, Rudek MA, Piekarz R (2017) Entinostat: a promising treatment option for patients with advanced breast cancer. Futur Oncol 13:1137-1148.

Cree BAC, Cutter G, Wolinsky JS, Freedman MS, Comi G, Giovannoni G, Hartung H-P, Arnold D, et al. (2020) Safety and efficacy of MD1003 (high-dose biotin) in patients with progressive multiple sclerosis (SPI2): a randomised, double-blind, placebocontrolled, phase 3 trial. Lancet Neurol 19:988-997.

Faraco G, Cavone L, Chiarugi A (2011) The therapeutic potential of HDAC inhibitors in the treatment of multiple sclerosis. Mol Med $17: 442-447$.

Faraco G, Pittelli M, Cavone L, Fossati S, Porcu M, Mascagni P, Fossati G, Moroni F, et al. (2009) Histone deacetylase (HDAC) inhibitors reduce the glial inflammatory response in vitro and in vivo. Neurobiol Dis 36:269-279.

Friday BB, Anderson SK, Buckner J, Yu C, Giannini C, Geoffroy F, Schwerkoske J, Mazurczak M, et al. (2012) Phase II trial of vorinostat in combination with bortezomib in recurrent glioblastoma: a north central cancer treatment group study. Neuro Oncol 14:215-221.

Galanis E, Jaeckle KA, Maurer MJ, Reid JM, Ames MM, Hardwick JS, Reilly JF, Loboda A, et al. (2009) Phase II trial of vorinostat in recurrent glioblastoma multiforme: a North Central Cancer Treatment Group Study. J Clin Oncol 27:2052-2058.

Ge Z, Da Y, Xue Z, Zhang K, Zhuang H, Peng M, Li Y, Li W, et al. (2013) Vorinostat, a histone deacetylase inhibitor, suppresses dendritic cell function and ameliorates experimental autoimmune encephalomyelitis. Exp Neurol 241:56-66.

Göschl L, Preglej T, Hamminger P, Bonelli M, Andersen L, Boucheron N, Gülich AF, Müller L, et al. (2018) A T cell-specific deletion of HDAC1 protects against experimental autoimmune encephalomyelitis. J Autoimmun 86:51-61.

Gray SG, Dangond F (2006) Rationale for the use of histone deacetylase inhibitors as a dual therapeutic modality in multiple sclerosis. Epigenetics 1:67-75.

Jayaraman A, Sharma M, Prabhakar B, Holterman M, Jayaraman S (2018) Amelioration of progressive autoimmune encephalomyelitis by epigenetic regulation involves selective repression of mature neutrophils during the preclinical phase. Exp Neurol 304:14-20.

Jayaraman A, Soni A, Prabhakar BS, Holterman M, Jayaraman S (2017) The epigenetic drug Trichostatin A ameliorates experimental autoimmune encephalomyelitis via $T$ cell tolerance induction and impaired influx of $\mathrm{T}$ cells into the spinal cord. Neurobiol Dis 108:1-12. 
Kappos L, Bar-Or A, Cree BAC, Fox RJ, Giovannoni G, Gold R, Vermersch P, Arnold DL, et al. (2018) Siponimod versus placebo in secondary progressive multiple sclerosis (EXPAND): a doubleblind, randomised, phase 3 study. Lancet 391:1263-1273.

KhorshidAhmad T, Acosta C, Cortes C, Lakowski TM, Gangadaran S, Namaka M (2016) Transcriptional regulation of brain-derived neurotrophic factor (BDNF) by methyl $\mathrm{CpG}$ binding protein 2 (MeCP2): a novel mechanism for re-myelination and/or myelin repair involved in the treatment of multiple sclerosis (MS). Mol Neurobiol 53:1092-1107.

Kutzelnigg A, Lassmann H (2014) Pathology of multiple sclerosis and related inflammatory demyelinating diseases. In: Handbook of Clinical Neurology. p. 15-58.

Laubach JP, Moreau P, San-Miguel JF, Richardson PG (2015) Panobinostat for the treatment of multiple myeloma. Clin Cancer Res 21:4767-4773.

Li S, Fossati G, Marchetti C, Modena D, Pozzi P, Reznikov LL, Moras ML, Azam T, et al. (2015) Specific inhibition of histone deacetylase 8 reduces gene expression and production of proinflammatory cytokines in vitro and in vivo. J Biol Chem 290:2368-2378.

Lillico R, Zhou T, Khorshid Ahmad T, Stesco N, Gozda K, Truong J, Kong J, Lakowski TM, et al. (2018) Increased post-translational lysine acetylation of myelin basic protein is associated with peak neurological disability in a mouse experimental autoimmune encephalomyelitis model of multiple sclerosis. J Proteome Res 17:55-62.

LoPresti P (2019) The selective HDAC6 inhibitor ACY-738 impacts memory and disease regulation in an animal model of multiple sclerosis. Front Neurol 10:51-61.

Mascarenhas J, Marcellino BK, Lu M, Kremyanskaya M, Fabris F, Sandy L, Mehrotra M, Houldsworth J, et al. (2020) A phase I study of panobinostat and ruxolitinib in patients with primary myelofibrosis (PMF) and post-polycythemia vera/essential thrombocythemia myelofibrosis (post-PV/ET MF). Leuk Res 88 106272.

Montalban X, Hauser SL, Kappos L, Arnold DL, Bar-Or A, Comi G, de Seze J, Giovannoni G, et al. (2017) Ocrelizumab versus placebo in primary progressive multiple sclerosis. $N$ Engl J Med 376:209-220.

Moreira JMA, Scheipers P, Sørensen P (2003) The histone deacetylase inhibitor Trichostatin A modulates CD4 $+\mathrm{T}$ cell responses. BMC Cancer 3:30.

Ocio EM, Vilanova D, Atadja P, Maiso P, Crusoe E, FernandezLazaro D, Garayoa M, San-Segundo L, et al. (2010) In vitro and in vivo rationale for the triple combination of panobinostat
(LBH589) and dexamethasone with either bortezomib or lenalidomide in multiple myeloma. Haematologica 95:794-803.

Ontaneda D, Tallantyre E, Kalincik T, Planchon SM, Evangelou N (2019) Early highly effective versus escalation treatment approaches in relapsing multiple sclerosis. Lancet Neurol 18:973-980.

Orillion A, Hashimoto A, Damayanti N, Shen L, Adelaiye-Ogala R, Arisa S, Chintala S, Ordentlich P, et al. (2017) Entinostat neutralizes myeloid-derived suppressor cells and enhances the antitumor effect of PD-1 inhibition in murine models of lung and renal cell carcinoma. Clin Cancer Res 23:5187-5201.

Peedicayil J (2016) Epigenetic drugs for multiple sclerosis. Curr Neuropharmacol 14:3-9.

Regna NL, Chafin CB, Hammond SE, Puthiyaveetil AG, Caudell DL, Reilly CM (2014) Class I and II histone deacetylase inhibition by ITF2357 reduces SLE pathogenesis in vivo. Clin Immunol 151:29-42.

Singh A, Bishayee A, Pandey A (2018) Targeting histone deacetylases with natural and synthetic agents: an emerging anticancer strategy. Nutrients 10:731.

Sun L, Telles E, Karl M, Cheng F, Luetteke N, Sotomayor EM, Miller RH, Seto E (2018) Loss of HDAC11 ameliorates clinical symptoms in a multiple sclerosis mouse model. Life Sci Alliance 1 e201800039.

Thompson AJ, Baranzini SE, Geurts J, Hemmer B, Ciccarelli O (2018) Multiple sclerosis. Lancet 391:1622-1636.

Trapp BD, Stys PK (2009) Virtual hypoxia and chronic necrosis of demyelinated axons in multiple sclerosis. Lancet Neurol 8:280-291.

Weiner HL (2008) A shift from adaptive to innate immunity: a potential mechanism of disease progression in multiple sclerosis. J Neurol 255:3-11.

Wössner N, Alhalabi Z, González J, Swyter S, Gan J, Schmidtkunz K, Zhang L, Vaquero A, et al. (2020) Sirtuin 1 inhibiting thiocyanates (S1th) - a new class of isotype selective inhibitors of NAD + dependent lysine deacetylases. Front Oncol 10:657.

Xie R, Li Y, Tang P, Yuan Q (2018) Design, synthesis and biological evaluation of novel 2-aminobenzamides containing dithiocarbamate moiety as histone deacetylase inhibitors and potent antitumor agents. Eur J Med Chem 143:320-333.

\section{APPENDIX A. SUPPLEMENTARY DATA}

Supplementary data to this article can be found online at https://doi.org/10.1016/j. neuroscience.2021.04.002. 